\title{
El arte relacional como herramienta en la gestión de públicos en los museos de Lima
}

\section{RELATIONAL ART AS A TOOL IN PUBLIC MANAGEMENT IN LIMA MUSEUMS}

Liliana Chaparro Huauya

Pontificia Universidad Católica del Perú

\section{RESUMEN}

En los años noventa, los expertos en la investigación artística comienzan a abordar la estética relacional a partir de los estudios del esteta francés Nicolas Bourriaud. Uno de los principios más importantes de esta corriente estética es que el objeto de estudio del arte no se concentre en el objeto artístico, sino en el vínculo que se genera entre el objeto artístico y el público, considerando la estrecha relación entre el sujeto, el objeto (obra de arte) y el artista. Estas ideas se gestan a partir de preceptos como los de Pierre Bourdieu, en los que señala que el arte actual es un espacio de relaciones objetivas entre posiciones, de lo cual se desprende, asimismo, que la obra artística funciona como un dispositivo relacional. Este artículo se basa en un plan de gestión de museos a través del arte relacional, entendido como una herramienta para mejorar las relaciones entre la comunidad y hacer de esta un miembro activo del museo. El arte relacional (mediante propuestas como la performance, el arte y la improvisación) ha brindado en los últimos años herramientas que capacitan al trabajador cultural para desarrollar posteriormente programas educativos, talleres y exposiciones que contemplen no solo al artista, al crítico de arte y al estudiante de historia de arte, sino también al público en general.

PALABRAS CLAVE: arte relacional, estética, museos, estudios, Perú, Lima, gestión de públicos

\section{ABSTRACT}

In the nineties, experts in artistic research began to address relational aesthetics from the studies of the French esthete Nicolas Bourriaud. One of the most important principles of this aesthetic current is that the object of study of art do not focus on the artistic object, but on the bond that is generated between the artistic object and the public; thus gaining a close relationship between the subject, object (work of art) and artist. These ideas are gestated from precepts such as those of Pierre Bourdieu in which he indicates that current art is a space of objective relationships between positions, which also shows that the artistic work functions as a relational device. This article is based on a museum management proposal through the use of Relational Art, understood as a tool to improve relations between the community and make them active members of the museum. Relational art (through proposals such as performance, art and improvisation) has provided, in the last years, tools that enable the cultural worker to subsequently develop educational programs, workshops, exhibitions that contemplate not only the artist but also the art critic and art history student; but to the general public.

KEYWORDS: relational art, aesthetic, museums, studies, Peru, Lima, public management 


\section{INTRODUCCIÓN}

En los últimos años, las industrias artísticas, la publicidad, la educación y otras esferas desarrollan la importancia del vínculo con el público dentro de sus estrategias y propuestas. En el campo de la educación se habla, por ejemplo, de educación disruptiva como una incursión en nuevas propuestas de enseñanza que vinculen de otra manera al alumno con el uso de tecnologías y nuevas conexiones con otras personas y sistemas. Esta tendencia responde a una constante necesidad de descubrir siempre nuevas maneras de vinculación con nuestro entorno.

Si miramos en retrospectiva unos años atrás, en los noventa, el esteta francés Nicolas Bourriaud (2006) propuso que el vínculo entre obra de arte, artista y público debe estrecharse más para un mejor entendimiento. Este punto de partida en la generación de un vínculo en el arte es lo que se define como arte relacional.

Pero ¿qué es el arte relacional? Es una expresión de los principios de la estética relacional que plantea Bourriaud en el libro que lleva el mismo nombre. Se reduce a la búsqueda de nuevas estrategias y/o prácticas artísticas que impliquen un vínculo más directo entre artista y público. Este artículo describe la aplicación de estos principios en el campo de la museología actual en nuestro país, con la finalidad de demostrar que el arte relacional puede ser un instrumento que permita a los museos desarrollar estrategias educativas y/o publicitarias que impliquen la aplicación del vínculo con el público y, por consiguiente, incrementar la afluencia de visitantes.

\section{LA IMPORTANCIA DE GENERAR ESPACIOS DE REFLEXIÓN MEDIANTE EL ARTE}

Tolosa Rivera (2015) sostiene la importancia del arte para la construcción de ciudadanía $y$, por ende, de un sistema de paz en un espacio de conflicto que dura por muchos años, mediante la inserción del arte en programas educativos locales. En ese sentido, el arte relacional es también una herramienta que permite desarrollar propuestas museográficas y que vinculan más al público dentro de un marco de construcción de paz, así como de ciudadanía.

De allí la importancia en generar espacios desde la sociedad civil en los que sea posible compartir conocimiento y saberes en torno a las experiencias a nivel personal y comunitario, que, en el marco del conflicto armado o fuera de este, son relevantes para hacer memoria de lo que somos como país, de los acontecimientos importantes de recordar y a su vez de cuál es la importancia de ello para la construcción de paz y transformación de conflictos. (Tolosa Rivera, 2015, p. 7)

El objetivo general de este artículo es demostrar que el arte relacional en los últimos años se ha insertado en el contexto limeño de los museos como una herramienta que permite desarrollar propuestas museográficas o educativas que implican la conexión, 
el vínculo con el otro y la experiencia de usuario, a los cuales llamaremos experiencias relacionales.

\section{¿Y CÓMO SE PUEDE APLICAR EL ARTE RELACIONAL EN LOS MUSEOS?}

Según Sábate y Gort (2012), los museos son espacios que deben orientar su continuidad al intercambio de un diálogo directo entre comunidad e institución. Específicamente el museo comunitario debe incidir en desarrollar estrategias que permitan al público relacionarse con los objetos expuestos: "La consecución de un museo comunitario debe basarse en el establecimiento de los mecanismos que lo hagan permeable a la sociedad que lo acoge y sostiene" (p. 18).

Una de las propuestas que el arte relacional considera para desarrollar estrategias que permitan al público acercarse a los objetos o discursos museográficos son los ejercicios o experiencias performáticas. Podemos mencionar trabajos de artistas como Rirkrit Tiravanija y Marina Abramović, entre otros, quienes proponen la performance como acto político y social. Guillermo Gómez Peña, artista mexicano, indica que el performer es un escritor, un artista, pero que también teoriza el arte, la política y la cultura. En nuestro contexto, la performance ha encontrado un espacio para decir lo que no se puede decir fácilmente en un discurso convencional. Veamos lo sucedido hace pocos años con una muestra en el LUM de 36 artistas. La exposición Resistencia visual 1992, inaugurada en el 2017 en dicho lugar, generó tanta controversia que ocasionó la destitución del director del museo Guillermo Nugent. Al margen de que esta muestra no es de performance, lo potente de su discurso social dejó claro que estamos en un contexto en el que todavía se vive censuras en espacios donde la libertad de expresión no debería ser puesta en vulnerabilidad.

En el Perú, el ejercicio de la performance cobra la dimensión de ser parte de un grupo que navega a contracorriente dentro del ejercicio de dictadura que se haya podido vivir. Un estudio de Víctor Vich aborda el tema de la performance asociado a la participación colectiva y política al final de la dictadura fujimorista. Asimismo, es importante añadir que en los últimos años el uso de la performance ha permitido vincular más directamente como medio artístico al público con el objeto de arte, e inclusive al artista. Artistas como Guillermo Castrillón, Diana Collazos, Lorena Peña accionan y teorizan el ejercicio de la performance, así como instituciones como el Museo de Arte Contemporáneo (MAC) involucran también la práctica de la performance en sus exhibiciones, talleres y presentaciones. (Chaparro, 2017, p. 33)

En ese sentido, el arte relacional, tal cual lo menciona Bourdieu, puede funcionar como un dispositivo social que permita generar nuevas conexiones de comunicación e interacción con el público. Es una herramienta que puede desarrollar y comunicar discursos sociales, así como construir identidad y ciudadanía; por consiguiente, crea interés y expectativa en el usuario, lo que incrementa el flujo de visitantes y públicos. En el 
siguiente apartado, se plantea la propuesta del arte relacional como herramienta para la generación de nuevos públicos más participativos e involucrados en los museos de Lima, partiendo de casos como el Museo Central (MUCEN), el Museo de Arte Contemporáneo (MAC) y la Casa de la Literatura (CASLIT).

No se puede dejar de mencionar también la museografía del LUM (Lugar de la Memoria, la Tolerancia y la Inclusión Social) como un referente de trabajo que involucra un vínculo más directo con el público. El LUM ha apostado por acercarse a sus visitantes mediante una propuesta museográfica que pone en evidencia una conexión con los hechos sucedidos en el período de terrorismo, narrados de una manera que lleva al usuario a insertarse en la experiencia y las vivencias de ese momento de oscurantismo en nuestro país.

\section{CASOS DE MUSEOS Y ARTE RELACIONAL: MUCEN, CASLIT Y MAC}

El Museo Central (MUCEN) presenta nuevas propuestas de experiencias relacionales como \#Etiquetarte o el Museo Abre de Noche (propuesta que varios museos en Lima realizan para una mayor afluencia de públicos a fines de mes normalmente), las cuales en los últimos años han generado que esta institución mejore su imagen y su posicionamiento como museo de Lima.

Además de razones de posicionamiento y marketing, este cambio - planteado por la directora Ulla Holmquist- evidencia reinvención, diálogo y modernización. Soy lejano a esa idea del museo como lugar sacro, con iluminación dramática, política y sexualmente correcto, donde una exposición sea el único atractivo y lleno de prohibiciones. Por lo contrario, valoro los museos que son espacios profanos, donde multitudes de escolares circulan vigorosamente y donde se respira libertad. El MUCEN se está convirtiendo en un espacio vivo. ("David Flores-Hora: MUCEN", 2017)

Por su parte, la Casa de la Literatura (CASLIT) aborda propuestas museográficas que involucran un acercamiento a la mirada del visitante. Es relevante mencionar la experiencia del usuario en la lectura y mirada de una exposición que no solo está dirigida a contarnos algo, sino a que seamos parte de la experiencia. Una de las exposiciones que más podríamos destacar es la de Mario Vargas Llosa hace unos años, cuyo planteamiento museográfico invita al usuario a adentrarse en el mundo de la experiencia.

El Museo de Arte Contemporáneo (MAC) desarrolla no solo propuestas museográficas, sino también talleres educativos acordes con las necesidades del público, pero con la finalidad de estrechar vínculos con este. En tal sentido, el equipo de los programas educativos en esta institución señala la importancia que esta entidad le da a la formación de públicos mediante experiencias relacionales. 
Cabe mencionar que la necesidad que tuvieron en común estas tres instituciones fue la vinculación más directa con su público, ese público que vive por ese mismo espacio, pero que por diferentes motivos no se volvía parte de ese lugar ni se identificaba con él. En los tres casos, se ha logrado estrechar la relación con los visitantes gracias a estas propuestas de experiencias relacionales, con las que vienen formando un público más participativo, cuestionador e involucrado.

\section{GESTIÓN DE PÚBLICOS: IMPACTO Y REFLEXIÓN}

Este artículo es parte de un trabajo de investigación sobre el uso del arte relacional como recurso para incrementar la gestión de públicos en los museos de Lima. Las cifras que permitirán conocer con mayor detalle este postulado todavía están en procesamiento, pero desde ya se viene evaluando el trabajo cualitativo de los museos mencionados. Es importante destacar también el efecto de estas experiencias relacionales en la formación de nuevos públicos.

Finalmente, después de lo acontecido en el LUM en el 2017, queda más clara la necesidad de seguir desarrollando la capacidad crítica y analítica del usuario y consumidor del museo. Ya los nuevos conceptos definen al museo como un espacio educativo de reconstrucción de ciudadanía y sociedad. El concepto de este como lugar sacrosanto que guarda colecciones es anacrónico con nuestro tiempo y contexto. El arte relacional, mediante sus propuestas museográficas, educativas e inclusive publicitarias (entiéndase esto último en relación con la comunicación y el marketing), o gestión de públicos es una herramienta que no solo contribuye a mejorar la afluencia de espectadores, sino que forja capacidad crítica. Guste o no a muchos, los museos son un espacio que debe incorporar este tipo de experiencias para continuar trabajando memoria, identidad, ciudadanía y sobre todo paz.

\section{REFERENCIAS}

Belenguer, M. C., y Melendo, M. (2012). El presente de la estética relacional: hacia una crítica de la crítica. Calle 14. Revista de Investigación en el Campo del Arte, 6(8), 88-100. https://doi.org/10.14483/udistrital.jour.c14.2012.1.a08

Bourriaud, N. (2006). Estética relacional (traducción de C. Beceyro y S. Delgado). Buenos Aires: Adriana Hidalgo, editora.

Chaparro, L. (2017). El arte relacional como herramienta en las nuevas metodologías de enseñanza en cursos de Humanidades de alumnos de Diseño. Actas EDK: Anuario de Arte y Diseño, 1, 30-34. Recuperado de http://repositorio.usil.edu.pe/ bitstream/USIL/2637/3/2017_USIL_Actas-EDK.pdf 
David Flores-Hora: MUCEN [opinión]. (27 de julio del 2017). Perú21. Recuperado de https:// peru21.pe/cultura/david-flores-hora-mucen-opinion-90791-noticia/

Marxen, E. (2009). La etnografía desde el arte. Definiciones, bases teóricas y nuevos escenarios. Alteridades, 19(37), 7-22. Recuperado de http://www.scielo.org.mx/ scielo.php?script=sci_arttext\&pid=S0188-70172009000100002

Sábate, M., y Gort, R. (2012). Museo y comunidad. Un museo para todos los públicos. Barcelona: Ediciones Trea.

Taylor, D., y Fuentes, M. (2011). Estudios avanzados de performance. Ciudad de México: Fondo de Cultura Económica.

Tolosa Rivera, M. (2015). El arte como posible herramienta metodológica para la construcción de paz (trabajo de investigación para optar el título de especialista en acción sin daño y construcción de paz). Universidad Nacional de Colombia, Bogotá, Colombia. 